\title{
Neurogenic Changes in the Upper Airway of Patients with Obstructive Sleep Apnea
}

\author{
Julian P. Saboisky ${ }^{1,2}$, Daniel W. Stashuk ${ }^{3}$, Andrew Hamilton-Wright ${ }^{4}$, Andrea L. Carusona ${ }^{1}$, \\ Lisa M. Campana5, John Trinder6, Danny J. Eckert1,2, Amy S. Jordan6, David G. McSharry ${ }^{1,2}$, \\ David P. White ${ }^{2}$, Sanjeev Nandedkar7, William S. David ${ }^{2,8}$, and Atul Malhotra1,2
}

\begin{abstract}
${ }^{1}$ Sleep Disorders Program, Division of Sleep Medicine, Brigham and Women's Hospital, Boston, Massachusetts; ${ }^{2}$ Harvard Medical School, Boston, Massachusetts; ${ }^{3}$ Department of Systems Design Engineering, University of Waterloo, Waterloo, Ontario; ${ }^{4}$ Mathematics and Computer Science, Mount Allison University, Sackville, New Brunswick, Canada; ${ }^{5}$ Boston University, Boston, Massachusetts; ${ }^{6}$ Department of Psychological Sciences, University of Melbourne, Victoria, Australia; ${ }^{7}$ CareFusion (S.D.N.), Middleton, Wisconsin; and ${ }^{8}$ Department of Neurology, Massachusetts General Hospital, Boston, Massachusetts
\end{abstract}

Rationale: Controversy persists regarding the presence and importance of hypoglossal nerve dysfunction in obstructive sleep apnea (OSA).

Objectives: We assessed quantitative parameters related to motor unit potential (MUP) morphology derived from electromyographic (EMG) signals in patients with OSA versus control subjects and hypothesized that signs of neurogenic remodeling would be present in the patients with OSA.

Methods: Participants underwent diagnostic sleep studies to obtain apnea-hypopnea indices. Muscle activity was detected with $50-\mathrm{mm}$ concentric needle electrodes. The concentric needle was positioned at more than 10 independent sites per subject, after the local anatomy of the upper airway musculature was examined by ultrasonography. All activity was quantified with subjects awake, during supine eupneic breathing while wearing a nasal mask connected to a pneumotachograph. Genioglossus EMG signals were analyzed offline by automated software (DQEMG), which extracted motor unit potential trains (MUPTs) contributed by individual motor units from the composite EMG signals. Quantitative measurements of MUP templates, including duration, peak-to-peak amplitude, area, areato-amplitude ratio, and size index, were compared between the untreated patients with OSA and healthy control subjects.

Measurements and Main Results: A total of 1,655 MUPTs from patients with OSA ( $n=17 ; A H I, 55 \pm 6 / h)$ and control subjects $(n=14 ; A H I$, $4 \pm 1 / h$ ) were extracted from the genioglossus muscle EMG signals. MUP peak-to-peak amplitudes in the patients with OSA were not different compared with the control subjects $(397.5 \pm 9.0$ vs. $382.5 \pm 10.0 \mu \mathrm{V})$. However, the MUPs of the patients with OSA were longer in duration $(11.5 \pm 0.1$ vs. $10.3 \pm 0.1 \mathrm{~ms} ; P<0.001)$ and had a larger size index $(4.09 \pm 0.02$ vs. $3.92 \pm 0.02 ; P<0.001)$ compared with control subjects.

Conclusions: These results confirm and quantify the extent and existence of structural neural remodeling in OSA.

Keywords: motor unit; respiration; tongue; genioglossus; respiratory

(Received in original form June 17, 2011; accepted in final form October 3, 2011) Supported by NIH 5K24HL093218-02 and 5R01HL090897-02, AHA 0840159N, NIH R01 HL085188, and P01 HL 095491.

Author Contributions: Conception and design: J.P.S., J.T., L.M.C., S.N., D.J.E., A.S.J., and A.M.; analysis and interpretation: J.P.S., D.W.S., A.H.-W., S.N., A.L.C., and A.M.; drafting the manuscript for important intellectual content: J.P.S., D.W.S. A.H.-W., J.T., D.P.W., L.M.C., A.L.C., D.G.M., D.J.E., A.S.J., and S.N.

Correspondence and requests for reprints should be addressed to Julian Saboisky, Ph.D., Neuroscience Research Australia (NeuRA), Barker Street, Randwick, Sydney, NSW, 2031, Australia. E-mail: j.saboisky@neura.edu.au

This article has an online supplement, which is available from this issue's table of contents at www.atsjournals.org

Am J Respir Crit Care Med Vol 185, Iss. 3, pp 322-329, Feb 1, 2012

Copyright $\odot 2012$ by the American Thoracic Society

Originally Published in Press as DOI: 10.1164/rccm.201106-1058OC on October 20, 2011 Internet address: www.atsjournals.org

\section{AT A GLANCE COMMENTARY}

Scientific Knowledge on the Subject

Several studies have supported sensory and motor impairments in the upper airway of patients with obstructive sleep apnea. Complex interactions of vibration, inflammation, and hypoxia are reported to contribute. The structure, and innervation, of hypoglossal nerve activity to muscles of the upper airway may affect the neural drive, but is not fully understood.

What This Study Adds to the Field

Structural remodeling of the peripheral branches of the hypoglossal nerve that innervates the largest upper airway dilator muscle (genioglossus) occurs in obstructive sleep apnea. These alterations, collectively referred to as neurogenic changes, are likely to arise as a result of deinnervation, collateral sprouting, and reinnervation.

Controversy remains in the obstructive sleep apnea (OSA) field as to how upper airway neurogenic changes may relate to disease pathogenesis/progression $(1,2)$. Changes in nerve and muscle function may occur in the pharynx of patients with OSA as a result of the disease (e.g., vibration through snoring [3-5] or hypoxemia [6]), potentially contributing to diminished upper airway muscle function. The muscles of the upper airway including the genioglossus, palatopharyngeus, pharyngeal constrictors, and musculus uvulae in patients with OSA contain a high percentage of type II muscle fibers compared with non-OSA control subjects (7-12), a difference that may represent an adaptive response to mechanical strain and/or neuronal activity. Deeper extrinsic dilator muscles, such as the genioglossus, may also be affected by neurogenic alterations (13), possibly contributing to the observed increase in genioglossus activity ("compensatory neural drive") in patients with OSA (14).

Motor unit potential (MUP) durations are increased in neurogenic disorders and, thus, neuromuscular dysfunction can be assessed by MUP analysis (15-17). Increased MUP duration has been attributed to motor unit remodeling as a result of denervation, collateral sprouting, and reinnervation (18-20). Motor units that have reinnervated orphaned fibers will have greater numbers of muscle fibers, and thus typically produce MUPs with an increased area (21). By combining various electrophysiological techniques to study motor units $(17,22)$, both temporal and spatial resolution can be maximized to explore neuromuscular function in the 
TABLE 1. SUBJECT DEMOGRAPHICS FOR STUDY POPULATIONS

\begin{tabular}{lcc}
\hline Subject Characteristic & Control & OSA \\
\hline Subjects & 14 & 17 \\
Age, yr & $34.4 \pm 2.8$ & $42.3 \pm 2.4^{*}$ \\
Number of units & 700 & 955 \\
Female/male & $6 / 8$ & $2 / 15$ \\
Apnea-hypopnea index, events per hour of sleep & $3.7 \pm 0.7$ & $54.8 \pm 6.1^{*}$ \\
Height, cm & $169.9 \pm 3.2$ & $177.1 \pm 2.1$ \\
Weight, kg & $86.1 \pm 7.6$ & $100.7 \pm 4.5$ \\
Body mass index, kg/m ${ }^{2}$ & $29.4 \pm 1.9$ & $32.2 \pm 1.4$ \\
Neck size, cm & $38.4 \pm 1.2$ & $42.5 \pm 0.7^{*}$ \\
Number of units per subject & $50.0 \pm 4.8$ & $56.2 \pm 5.8$ \\
Inferior margin of geniohyoid muscle, mm & $14.5 \pm 1.0$ & $16.1 \pm 1.1$ \\
Inferior margin of genioglossus muscle, mm & $23.8 \pm 0.9$ & $26.8 \pm 1.0^{*}$ \\
Genioglossus width, mm & $17.8 \pm 0.6$ & $18.2 \pm 0.5$ \\
Modified Borg Scale score, 0-10 & $2.6 \pm 0.4$ & $2.1 \pm 0.2$ \\
\hline
\end{tabular}

Definition of abbreviation: OSA = obstructive sleep apnea.

The modified Borg Scale was used to measure the "worst level of pain" experienced by each subject during the recording, with $0=$ nothing at all and $10=$ maximal.

Values represent means \pm SEM, unless otherwise specified

* $P<0.05$.

upper airway. Whereas selective concentric needle electrodes assess the closest muscle fibers of a motor unit (providing local muscle fiber information [21, 23]), the large detection surface of a concentric needle macro (CNMACRO) electrode provides a more global view of the whole motor unit $(24,25)$ (see Figure E1 in the online supplement). Thus, in early stages of neuromuscular disorders such as amyotrophic lateral sclerosis and polio, MACRO MUPs typically increase in size, indicating compensatory reinnervation phenomena for the loss of motoneurons $(16,26,27)$. Further, the area of the CNMACRO MUP predicts the twitch force generated by the motor unit (28) and, therefore, provides unique insight into the contribution to the muscle force output from a single motor unit. Neuromuscular dysfunction may be important in sleep apnea if such changes contribute to impaired upper airway motor control, increased pharyngeal collapsibility, and/or muscle fatigue (4, 5, 9, 19, 29-37).

To assess the extent of neuromuscular changes in patients with OSA, we measured the morphology of populations of selective concentric and CNMACRO MUPs. We hypothesized that patients with OSA will have measureable evidence of collateral sprouting, manifested through a larger number of phases and turns yielding more complex potentials, in comparison with control subjects. Such findings could be indicative of reinnervation and potentially important in OSA pathogenesis, helping to define new therapeutic targets for OSA. Preliminary data have been presented as abstracts $(38,39)$.

\section{METHODS}

Further methodological detail is provided in the online supplement. Thirty-three people signed our institutional review board-approved consent form, 2 were excluded for technical reasons, and of the remainder, 17 had OSA and 14 were control subjects (demographics in Table 1). These recordings were all completed at the Brigham and Women's Hospital in Boston, Massachusetts and all subjects were recruited specifically for this investigation. All subjects were screened to exclude major medical and/or neurological disorders, other than OSA. The local anatomy of the upper airway musculature of each participant was examined by ultrasonography $(13,40)$ (Table 1$)$.

With the subjects relaxed and lying supine a small reference location was drawn $10 \mathrm{~mm}$ posterior to the genial tubercle, and the chin was thoroughly cleaned before the insertion of two concentric needle electrodes into the genioglossus $(>25.5 \mathrm{~mm})$. Under topical anesthetic, the first concentric needle was used as a CNMACRO electrode and was positioned approximately $10 \mathrm{~mm}$ beyond the genioglossus/geniohyoid aponeurosis, where it remained in position for the entire recording procedure; such that it had a constant recording length. The second selective concentric needle electrode was initially positioned to the same depth and in close proximity $(\sim 4 \mathrm{~mm})$ to the CNMACRO electrode, but was manipulated to detect MUPs at independent positions.

Concentric needle signals were filtered $(10 \mathrm{~Hz}$ to $10 \mathrm{kHz})$ and amplified $(\times 200 \mu \mathrm{V})($ Sapphire 2; TECA, Pleasantville, NY). The CNMACRO signals were detected with the cannula of the CNMACRO referenced to a remote surface electrode (24). The CNMACRO signals were filtered $(10 \mathrm{~Hz}$ to $6 \mathrm{kHz})$ and amplified $(\times 10 \mathrm{k})$. A ground was

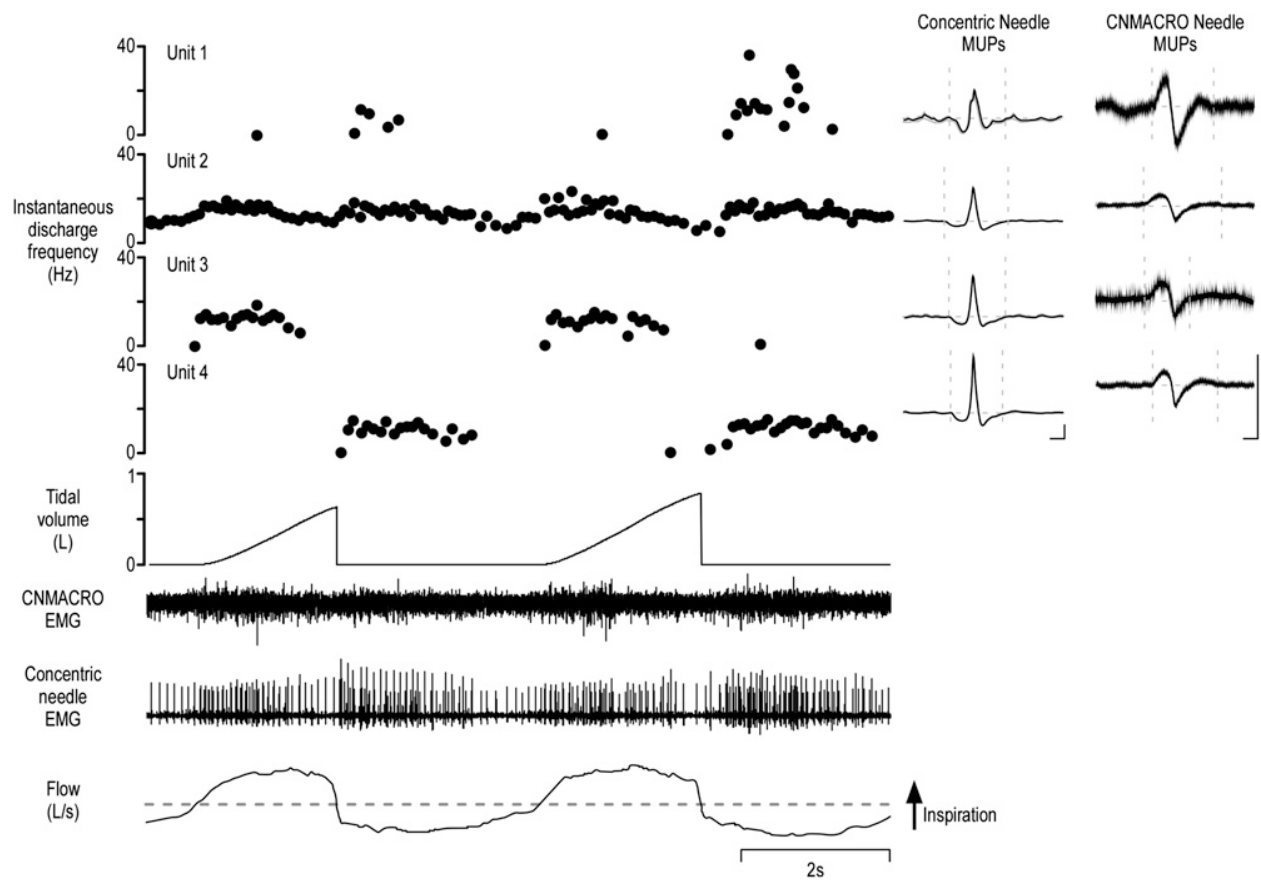

Figure 1. Typical example of genioglossus data with four motor units concurrently active during quiet breathing. Shown is a typical example of data extracted from a recording from a single subject. From bottom to top: respiratory airflow, selective concentric needle electromyography (EMG), concentric needle macro (CNMACRO) EMG (recorded from the cannula of the concentric needle), tidal volume, and the instantaneous discharge frequency of four single motor units extracted after the decomposition of the raw composite concentric needle EMG signal. Inset: The averaged selective concentric needle motor unit potential (MUP) templates and the ensemble averaged CNMACRO MUPs. Calibrations of the selective concentric needle MUPs and CNMACRO MUPs, $100 \mu \mathrm{V}$ and 2 milliseconds. 


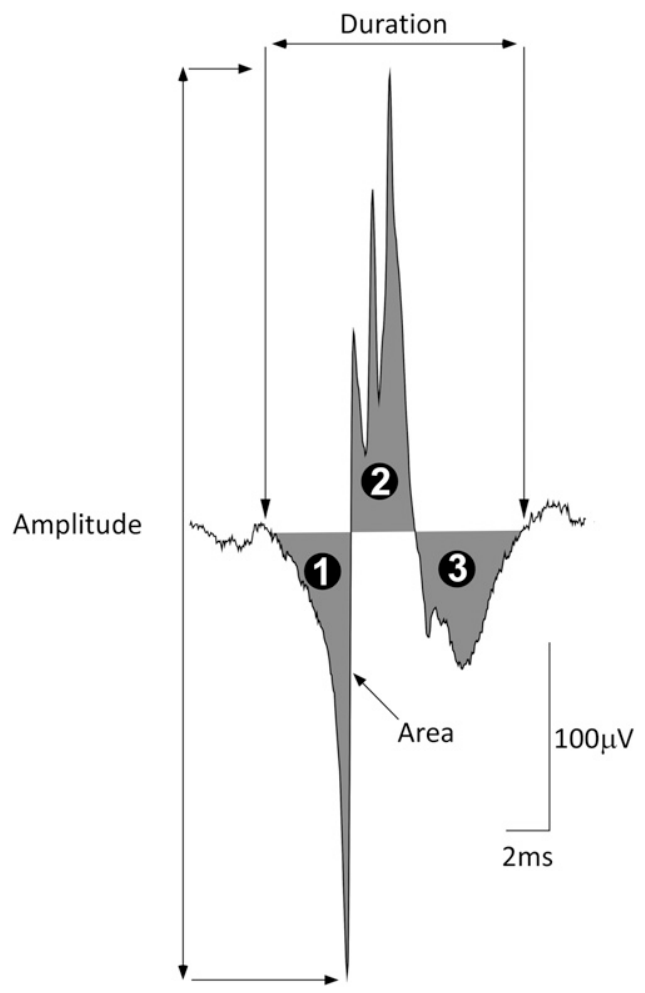

Figure 2. Neurophysiological features measured from the selective concentric needle motor unit potential templates. Selective concentric needle motor unit potential (MUP) analysis provides quantitative insight into the physiology of the motor unit. As such, it is possible to differentiate the normal muscle from neurogenic injury by MUP analysis. Global features included in the analysis were the duration of the motor unit potential as measured between the onset of the first and the offset of the last peak of the MUP (22). Duration is related to the number of muscle fibers contributing to the motor unit. Peak-to-peak amplitude is calculated as the voltage difference of the MUP and is determined by the diameter and number of muscle fibers closest to the electrode. Area is calculated by measuring the rectified MUP (over the duration). Area is less sensitive to measurement error than amplitude and is able to characterize atypical potentials. The number of phases (indicated by the black numbered circles) of the MUP are defined as the number of baseline crossings. The number of turns are defined as a change in the direction of the MUP waveform with a magnitude of at least $25 \mu \mathrm{V}$. To quantify further MUP complexity, a number of indices were used, including the following: area/phase ratio, which is better able to distinguish myopathic MUPs from normal MUPs than area alone. The area/amplitude ratio (63) and size index are parameters that assist in discriminating between neurogenic and normal MUPs and control for the influence of needle position (43). The relative irregularity coefficient (45) captures the complexity of the MUP by quantifying detailed features of the MUP and is calculated as follows: RIR $=[(\mathrm{S}-$ $2 \mathrm{~A}$ ) $/ 2 \mathrm{~A}] \times 100$ (where $\mathrm{A}$ is the amplitude, and $\mathrm{S}$ is calculated as the sum of the absolute values of the intersample changes seen in the MUP waveform template, i.e., the irregularity in the MUP template). Thus, with partial denervation there is structural remodeling with surviving axons projecting small braches ("collateral sprouting") to reinnervate muscle fibers denervated by the loss of axons. These new branches conduct impulses with a lower velocity over longer distances, reflected as more complex MUP waveforms. All parameter values were manually inspected by a single investigator using DQEMG and who was blinded to subject status. The structures of the concentric needle macro (CNMACRO) MUPs were also measured, using these reference points.

placed over the right clavicle. All electromyographic (EMG) signals were sampled at $25 \mathrm{kHz}$ (Spike2; CED, Cambridge, UK), and stored for offline analysis. During the procedure the subjects had documented
TABLE 2. MATCHED SUBJECT DEMOGRAPHICS: SUBANALYSIS FOR AGE- AND BODY MASS INDEX-MATCHED INDIVIDUALS

\begin{tabular}{lcc}
\hline Subject Characteristic & Control & OSA \\
\hline No. of subjects & 9 & 9 \\
Age, yr & $37.4 \pm 3.4$ & $37.7 \pm 3.8$ \\
Number of units & 475 & 420 \\
Female/male & $1 / 8$ & $1 / 8$ \\
Apnea-hypopnea index, events per hour of sleep & $4.2 \pm 1.0$ & $45.9 \pm 8.3^{*}$ \\
Height, cm & $175.8 \pm 3.3$ & $177.5 \pm 2.7$ \\
Weight, kg & $97.3 \pm 9.7$ & $95.0 \pm 3.4$ \\
Body mass index, kg/m ${ }^{2}$ & $31.2 \pm 2.5$ & $30.3 \pm 1.4$ \\
Neck size, cm & $41.1 \pm 0.9$ & $41.4 \pm 1.0$ \\
Number of units per subject & $52.8 \pm 6.7$ & $46.7 \pm 6.7$ \\
Inferior margin of geniohyoid muscle, mm & $14.1 \pm 1.2$ & $15.7 \pm 1.4$ \\
Inferior margin of genioglossus muscle, mm & $23.9 \pm 1.2$ & $26.5 \pm 1.2$ \\
Genioglossus width, mm & $18.3 \pm 0.5$ & $17.7 \pm 0.8$
\end{tabular}

Definition of abbreviation: OSA = obstructive sleep apnea.

Subanalysis included subjects who were matched for age, sex, and body mass index.

Values represent means \pm SEM, unless otherwise specified.

${ }^{*} P<0.05$

wakefulness as they breathed through a nasal mask attached to a pneumotachograph. All the recordings (Figure 1) were obtained without the subject's experiencing pain as measured on the basis of a 0 - to 10-point Borg Scale (13).

\section{Extraction of Single Motor Unit Activity}

Customized software (Spike2 scripts) converted the EMG signals into DQEMG format. Motor unit potential trains (MUPTs) were extracted from both the concentric needle EMG signals offline, using the DQEMG analysis system (version 3.4, revision 166) (41, 42). MUPs from EMG signals were decomposed on the basis of absolute threshold detection criteria (slope threshold, $0.3 \mathrm{~V} / \mathrm{s}$; amplitude threshold, $50 \mu \mathrm{V}$; time interval, $0.2 \mathrm{~ms}$ ). MUPs were averaged for each MUPT from both the selective concentric needle and CNMACRO signals. Measured MUP template features included the following: duration, amplitude, area, number of phases, and number of turns (see Figure 2). Calculations of area-to-amplitude ratio; size index (43), calculated as $2 \times$ $\log _{10}$ [amplitude $\left.(\mathrm{mV})\right]+$ [area/amplitude $\left.(\mathrm{ms})\right]$; and relative irregularity coefficient $(44,45)$, calculated as $\mathrm{RIR}=\{[\mathrm{S}-(2 \times$ amplitude $)] /(2 \times$ amplitude) $\} \times 100$ ( $\mathrm{S}$ is calculated as the absolute value of the intersample changes seen in the MUP template) were made. All MUP

\section{TABLE 3. KEY RESULTS FROM 31 SUBJECTS}

\begin{tabular}{lcc}
\hline Subject Characteristic & Control & OSA \\
\hline Number of units & 700 & 955 \\
Duration, $\mathrm{ms}$ & $10.3 \pm 0.1$ & $11.5 \pm 0.1^{*}$ \\
Amplitude, $\mu \mathrm{V}$ & $382.5 \pm 10.0$ & $397.5 \pm 9.0$ \\
Area, $\mu \mathrm{V} \cdot \mathrm{ms}$ & $514.6 \pm 13.3$ & $589.7 \pm 12.2^{*}$ \\
Phases & $2.8 \pm 0.03$ & $3.0 \pm 0.03^{*}$ \\
Turns & $3.6 \pm 0.06$ & $3.8 \pm 0.05^{*}$ \\
Area-to-amplitude ratio & $1.4 \pm 0.02$ & $1.6 \pm 0.02^{*}$ \\
Size index & $3.9 \pm 0.02$ & $4.1 \pm 0.02^{*}$ \\
Relative irregularity coefficient & $19.4 \pm 0.7$ & $25.9 \pm 0.7^{*}$ \\
Area/phase, $\mu \mathrm{V} \cdot \mathrm{ms}$ & $182.9 \pm 4.6$ & $203.1 \pm 4.3^{*}$ \\
MUPTs/contraction & $3.1 \pm 0.1$ & $3.5 \pm 0.1^{*}$ \\
Mean firing rate, Hz & $17.0 \pm 0.2$ & $17.7 \pm 0.2^{*}$ \\
Number of CNMACRO units & 446 & 668 \\
CNMACRO duration, ms & $13.0 \pm 0.3$ & $14.9 \pm 0.3^{*}$ \\
CNMACRO amplitude, $\mu \mathrm{V}$ & $42.9 \pm 2.0$ & $57.6 \pm 2.1^{*}$ \\
CNMACRO area, $\mu \mathrm{V} \cdot \mathrm{ms}$ & $106.2 \pm 4.4$ & $143.6 \pm 5.2^{*}$ \\
CNMACRO size index & $4.3 \pm 0.1$ & $4.3 \pm 0.1$ \\
\hline
\end{tabular}

Definition of abbreviations: CNMACRO = concentric needle macro; MUPTs = motor unit potential trains; OSA = obstructive sleep apnea.

Values represent means \pm SEM, unless otherwise specified.

* $P<0.05$ 

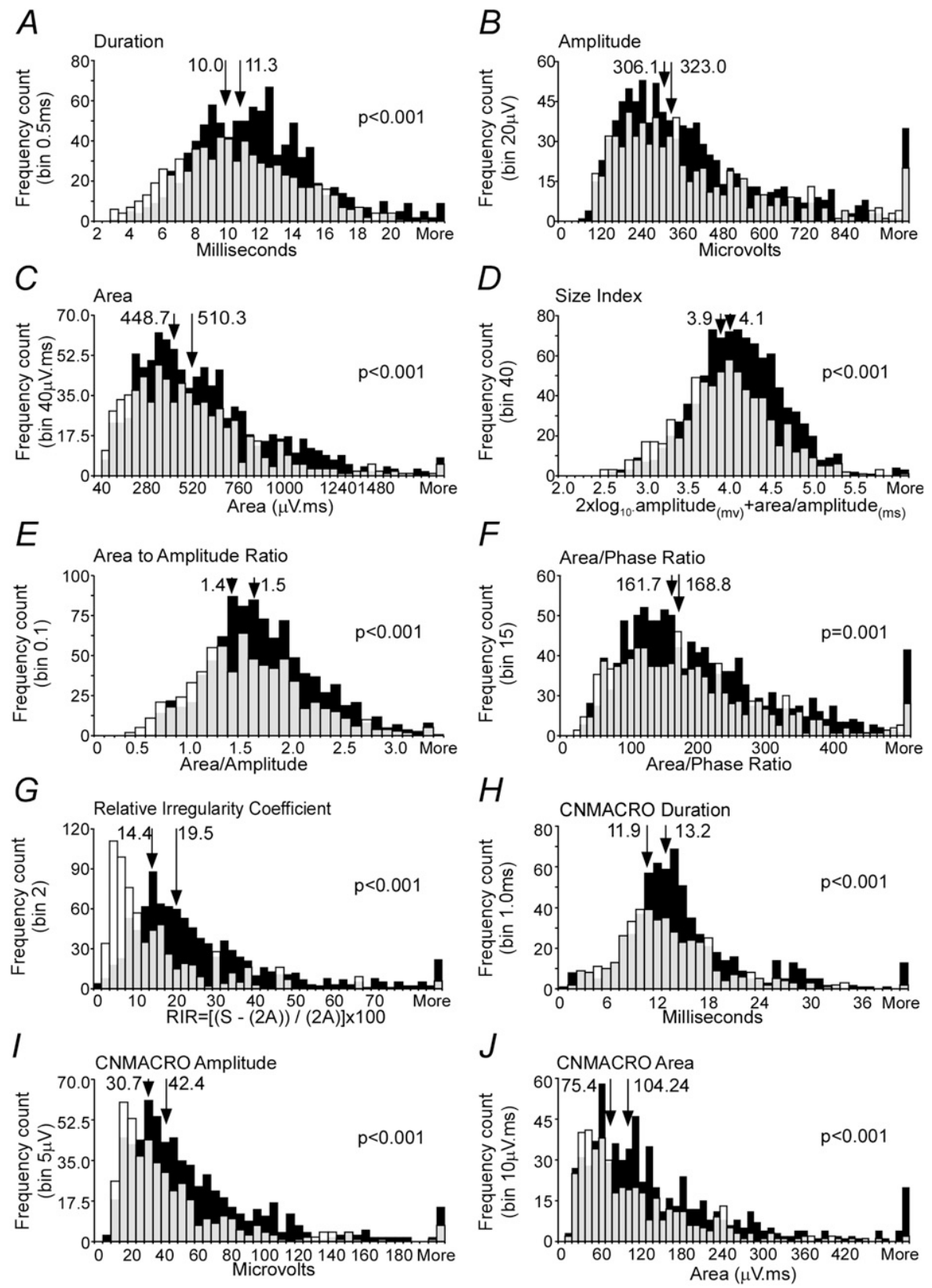

Figure 3. Measured and calculated variables from the selective concentric needle and concentric needle macro (CNMACRO) motor unit potentials (MUPs). Histogram display with the patients with obstructive sleep apnea (OSA) depicted in black and control subjects in white. Gray opacity depicts where there is overlap in the distribution of results for patients with OSA and control subjects. Medians are indicated by arrows in each panel, and in each circumstance the value for control subjects is at left and the distribution for patients with OSA is shifted to the right. $(A-G)$ The measured variables from selective concentric needle MUPs, including the following: $(A)$ duration, $(B)$ peak-to-peak amplitude, and $(C)$ area. $(D-G)$ The calculated variables from the concentric needle MUPs, including the following: size index calculated as $2 \times \log _{10}($ amplitude $(\mathrm{mV}))+$ (area/amplitude $(\mathrm{ms}))(D)$, area to amplitude ratio $(E)$, area/ phase ratio $(F)$, and relative irregularity coefficient $(G)$. (H- $)$ The measured variables from CNMACRO MUPs, including the following: $(H)$ duration, (I) amplitude, and $(I)$ area. Significance is given where appropriate in the respective panels. feature values of all the subjects were evaluated by a single investigator in accordance with standardized criteria (22).

A subanalysis was performed on nine patients with OSA and nine control subjects (Table 2) matched for age, sex, and body mass index (BMI). Statistical differences were assessed by one-way analysis of variance (ANOVA) and, when data were not normally distributed, then Kruskal-Wallis ANOVA on rank was performed with Dunn's method post-hoc analysis. Statistical significance was set at $P<0.05$. Values are given as means \pm SEM unless otherwise specified.

\section{RESULTS}

\section{Total Analysis}

Data were extracted from 31 subjects, yielding a total of 503 successful unitary recordings from the genioglossus muscle with concentric needle and CNMACRO electrodes (Table 1). From these detected signals a total of 1,655 valid MUPTs were extracted from the selective concentric needle (OSA, 955; control subjects, 700), and 1,114 CNMACRO MUPs were identified with the aid of DQEMG (OSA, 668; control subjects, 446). On average, the number of MUPTs extracted per recording was higher for the patients with OSA compared with the control subjects $(3.4 \pm 0.1$ vs. $3.1 \pm 0.1 ; P<0.05$, Dunn's method).

\section{Motor Unit Potentials}

All MUPTs extracted from selective concentric needle EMG signals were detected during normal eupneic breathing. An example of a typical recording is shown in Figure 1. Selective concentric needle MUPs detected in the patients with OSA had longer durations, greater area, and larger size index, areato-amplitude ratio, and relative irregularity ratio, but not greater amplitude, than control subjects (see Table 3, Figures $3,4 A-F$, and 5; and Supplementary Table E1 for one value/ 

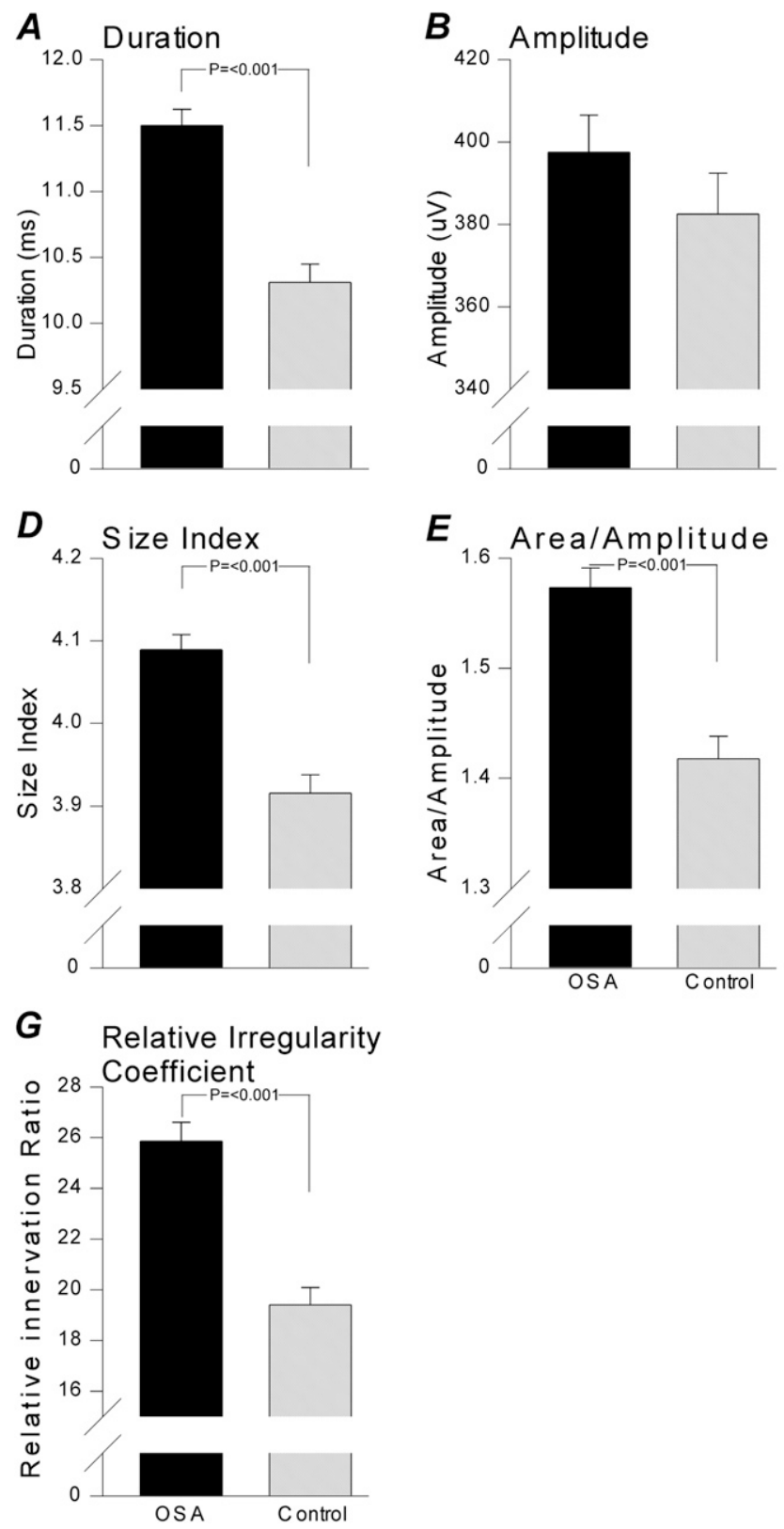

subject). Although the initial aims of the study did not include age as a factor, age was retrospectively studied. We did not observe an interaction between age and groups (Figure E1). The more complex MUPs, measured on the basis of the relative irregularity, were detected in the patients with OSA and an association was found with minimal oxygen saturation detected from the overnight polysomnography examination $(r=-0.639$, $P=0.0002$; Figure 6).

\section{CNMACRO Motor Unit Potentials}

The CNMACRO MUPs are more sensitive than selective concentric MUPs to changes in the total number of muscle fibers than to local reorganization of muscle fibers within a motor unit (46). Therefore, they can be used to measure reinnervation in terms of the number of active muscle fibers in a motor unit. CNMACRO potentials resemble a small surface-detected motor response. An example of a typical recording is shown in Figure 1. All CNMACRO MUPTs were estimated by triggeraveraging the cannula (CNMACRO needle electrode was
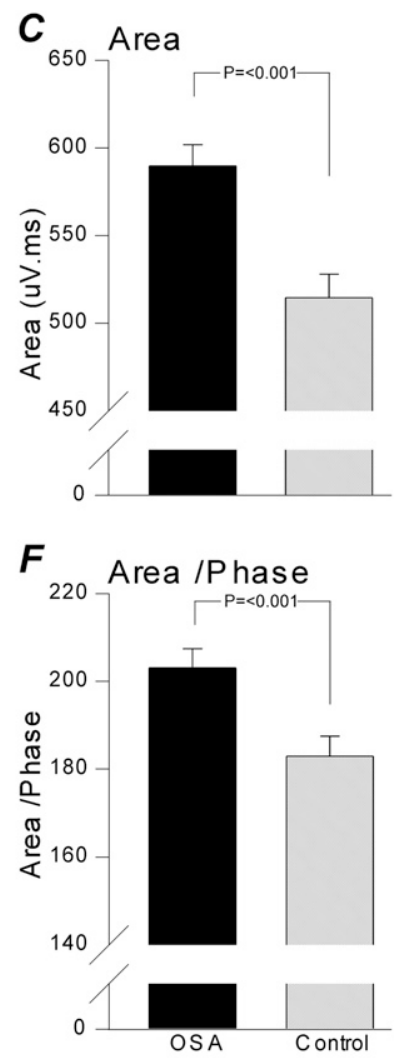

Figure 4. Mean measured and calculated variables from the concentric needle motor unit potentials. Each panel represents key averaged variables measured and calculated from all the individual concentric needle motor unit potentials (MUPs). The solid columns depict patient with obstructive sleep apnea (OSA) data and the shaded columns represent control subject data. Significance is given where appropriate in the respective panels. maintained at a constant depth of approximately $10 \mathrm{~mm}$, based on ultrasound measures)-detected EMG signals at the times of individual motor unit discharges during normal eupneic breathing. Not all sites that detected an MUP had an adequately measureable CNMACRO MUP. CNMACRO MUPs detected in the patients with OSA had longer durations, larger amplitudes, and greater areas than those detected in control subjects (Figure $3 G-I$ and Figure 5).

\section{Subanalysis of Matched Groups}

To control for the variation in age between the patients with OSA and control subjects, a subanalysis was performed on nine age-, sex-, and BMI-matched subjects (see Table 2 for demographic properties). This analysis included 420 MUPs from patients with OSA and 475 from control subjects (for results see Table 4; and Table E2 for one value/subject). In this analysis selective concentric needle MUPs of the patients with OSA had a longer duration, greater area, larger amplitude ratio, larger size index, and greater area-to-amplitude ratio, but not greater 

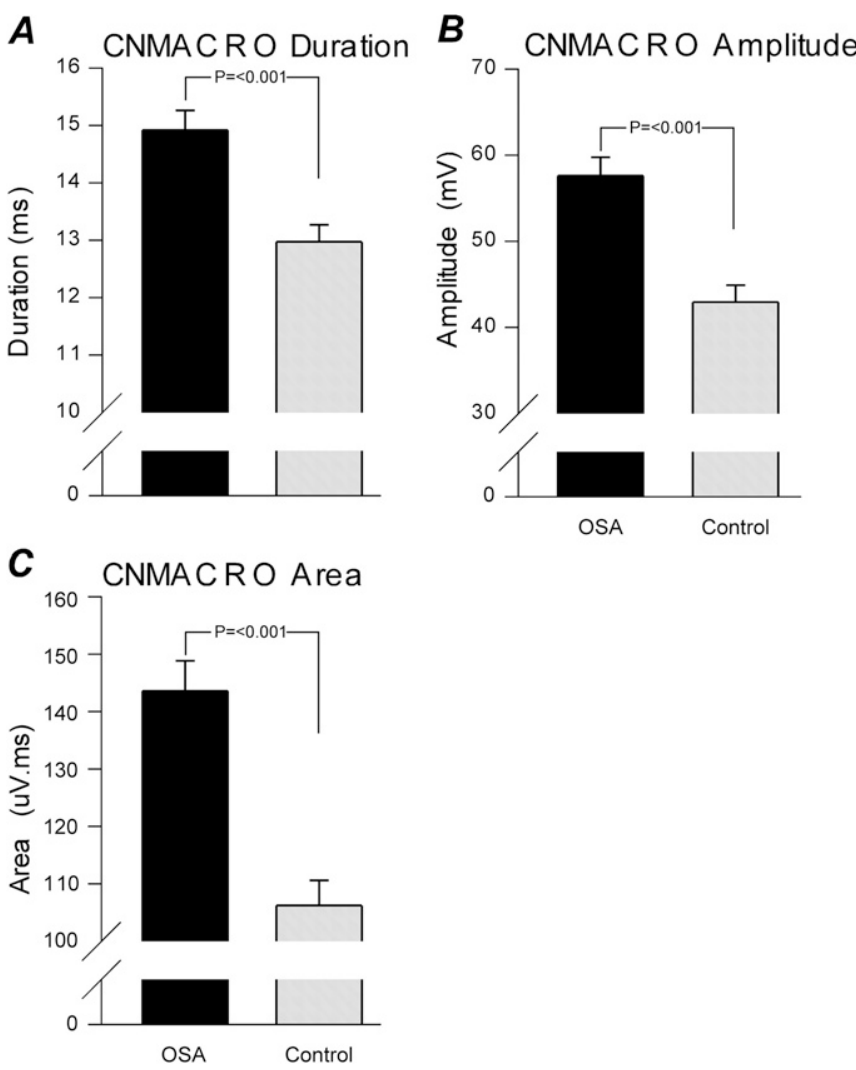

Figure 5. Mean measured variables from the concentric needle macro (CNMACRO) motor unit potentials (MUPs). Each panel represents key averaged variables measured and calculated from all the individual CNMACRO MUPs. The solid columns depict patient with obstructive sleep apnea (OSA) data and the shaded columns represent the control subject data. Significance is given where appropriate in the respective panels.

amplitude compared with the control subjects. The average number of MUPTs per recording was not larger in the patients with OSA compared with the matched control subjects. Consistent with the larger group comparison, CNMACRO MUPs detected in the matched patients with OSA resemble neurogenic MUPs with larger amplitudes (47) and greater indices than those detected in control subjects (Table 4).

\section{DISCUSSION}

Our study supports the hypothesis that patients with OSA have neurogenic changes in the genioglossus. We found MUP waveforms with increased durations, areas, and complexities, suggesting the peripheral axons have sprouted to adjust to changes in physiological requirements. These findings are similar to those seen in neuropathies, for example, vibration-induced injuries $(48,49)$. These data strongly indicate the presence of "neurogenic" changes in OSA $(6,10,11,13,19,30-32,36,50-52)$.

Established EMG techniques are available to study subtle changes in motor units $(17,22)$. We combined two techniques: (1) selective concentric needle electrodes to detect primarily from the closest muscle fibers of a motor unit, together with (2) the nonselective recordings of concentric needle macro (CNMACRO) electrodes, to assess the density and distribution of the whole cross-section of a motor unit $(21,23)$. For diagnosing neuromuscular disorders (15), MUP measurements are reliable indicators that have been well validated by histology (53). Our selective concentric needle data show evidence for

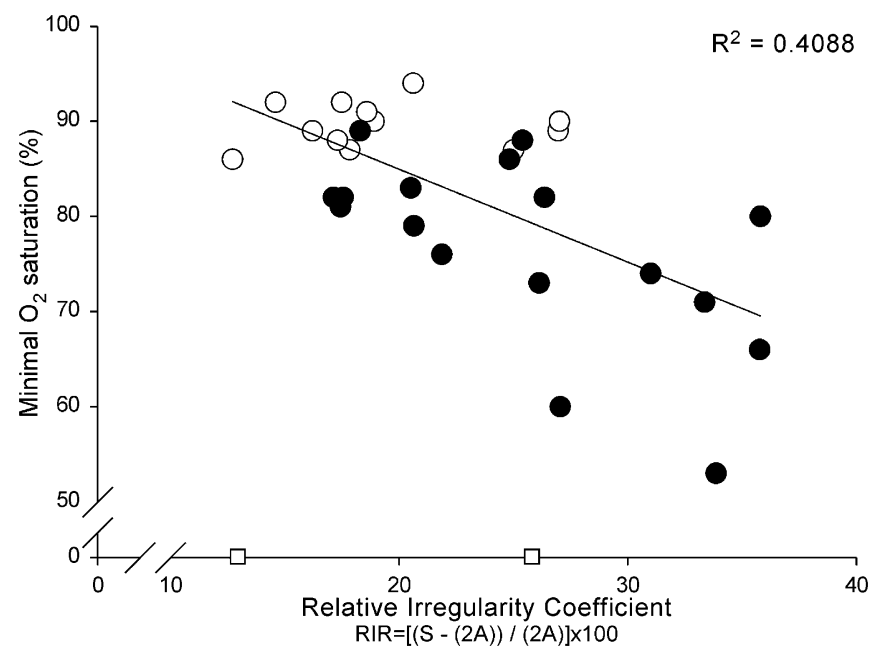

Figure 6. Mean relative irregularity coefficient versus minimal oxygen saturation. More complex motor unit potentials (MUPs), as calculated with the relative irregularity, are associated with minimal oxygen saturation detected from the overnight polysomnography examination. The Pearson correlation coefficient was calculated from the mean value of 29 subjects $(r=-0.639 ; P=0.000188)$. The open circles depict the healthy control subjects while solid circles depict the untreated patients with obstructive sleep apnea. The two open squares on the $x$ axis represent two control subjects from whom minimal oxygen data were not obtained (not included in the correlation).

a $14 \%$ increase in MUP duration, a finding consistent with reinnervation due to an increased number of fibers innervated by a single axon $(18-20,54)$. This finding is supported by Boyd and colleagues (30), who elegantly demonstrated an overexpression of neural cell adhesion molecule protein in palatal muscles of patients with OSA, a finding that implies active collateral sprouting. Furthermore, we found that the complexity of the MUP waveforms (as assessed by the relative irregularity coefficient $[44,45]$, i.e., normalized length of MUP) to be increased by $33 \%$ in patients with OSA and to correlate with minimal oxygen desaturations (see Figure 6). The OSA compensatory neural drive hypothesis is supported by evidence that the amplitude of the multiunit EMG is increased in patients with OSA (14). As the multiunit electromyogram is dependent on the number and complexity of MUPs it is often used as an estimate of the neural drive to the muscle. Genioglossus multiunit EMG amplitude corresponds to the sum of MUPs. Thus, changes in the global features of MUPs (see Figure 2) can influence motor unit potential summation and modulate EMG amplitude independent of the output from the hypoglossal nerve (e.g., Reference 53). Direct associations connecting MACRO MUP size and muscle force have been previously established $(25,27,28$, 55 ). Thus, the increased CNMACRO potentials (see Figure 5) may indicate that the genioglossus motor units in patients with OSA have increased force generation per activation. The observed increase in MUPs is also consistent with previous investigations that have shown histological differences in muscle fiber types in snorers and patients with OSA $(7-9,11,12,56)$. Thus, genioglossus muscle fibers in patients with OSA may have the ability to produce higher twitch forces, although possibly at the expense of longer recovery times, that is, fatigability $(9,57,58)$.

The cross-sectional design of this study means that it is not possible to identify the causative nature of this remodeling. Although it is likely that neurogenic changes are due to the mechanical strain induced by tissue vibration (48), negative pharyngeal pressure swings may also result in eccentric muscle contractions causing 
TABLE 4. KEY RESULTS FROM SUBANALYSIS OF NINE MATCHED SUBJECTS

\begin{tabular}{lcc}
\hline Subject Characteristic & Control & OSA \\
\hline Number of units & 475 & 420 \\
Duration, $\mathrm{ms}$ & $10.18 \pm 0.16$ & $11.62 \pm 0.17^{*}$ \\
Amplitude, $\mu \mathrm{V}$ & $390.33 \pm 12.40$ & $377.67 \pm 12.56$ \\
Area, $\mu \mathrm{V} \cdot \mathrm{ms}$ & $525.77 \pm 16.01$ & $595.76 \pm 18.23^{*}$ \\
Phases & $2.87 \pm 0.03$ & $2.99 \pm 0.04$ \\
Turns & $3.61 \pm 0.07$ & $3.71 \pm 0.07$ \\
Area-to-amplitude ratio & $1.44 \pm 0.02$ & $1.64 \pm 0.03^{*}$ \\
Size index & $3.94 \pm 0.03$ & $4.14 \pm 0.03^{*}$ \\
Relative irregularity coefficient & $20.43 \pm 0.87$ & $25.38 \pm 0.92^{*}$ \\
Area/phase & $186.20 \pm 5.60$ & $204.84 \pm 6.45^{*}$ \\
MUPTs/contraction & $3.4 \pm 0.19$ & $3.5 \pm 0.18$ \\
Mean firing rate, Hz & $18.0 \pm 0.26$ & $16.8 \pm 0.28^{*}$ \\
Number of CNMACRO units & 288 & 322 \\
CNMACRO duration, ms & $12.69 \pm 0.38$ & $13.30 \pm 0.54$ \\
CNMACRO amplitude, $\mu \mathrm{V}$ & $42.60 \pm 2.39$ & $47.96 \pm 2.56^{*}$ \\
CNMACRO area, $\mu \mathrm{V} \cdot \mathrm{ms}$ & $107.58 \pm 5.37$ & $112.05 \pm 7.34$ \\
CNMACRO size index & $4.5 \pm 0.13$ & $3.9 \pm 0.10^{*}$ \\
\hline
\end{tabular}

Definition of abbreviations: CNMACRO = concentric needle macro; MUPTs = motor unit potential trains; OSA = obstructive sleep apnea.

Values represent means \pm SEM, unless otherwise specified.

* $P<0.05$.

peripheral nerve damage. We speculate that this damage is likely to occur in the presence of hypoxemia, as has been demonstrated in nerve conduction studies (6). Investigation into the role of neuromuscular dysfunction in patients with OSA remains challenging, as needle biopsies are invasive and the upper airway dilator muscles are difficult to isolate.

Our study has strengths including the use of ultrasound guidance to ensure genioglossus placement of electrodes, in contrast to prior studies $(59,60)$. In addition, we argue that studying the muscle during eupneic breathing is of the utmost importance in terms of relevance to OSA, in contrast to recording ballistic voluntary tasks as is conventional in diagnostic EMG $(59,60)$. Last, we assessed a large unbiased sample of motor units in a large number of patients and thus believe we have performed the most thorough electrophysiological evaluation of the upper airway in humans to date.

Despite these strengths, we acknowledge a number of weaknesses. First, our groups were incompletely matched for age, sex, and BMI, although our findings were similar when using an age-, sex-, and weight-matched subset. Thus, we do not expect these factors to confound the results significantly. Third, we were unable to obtain measurements before and after therapy in our patients, which may yield valuable insight into the potential reversibility of the changes observed in future studies. However, we regard the electrophysiological methods used to be robust and well validated $(42,43,61,62)$ and less susceptible to sampling bias than biopsy in humans. Despite these acknowledged limitations, we believe our findings provide an important advance in the literature.

In summary, the results of this study indicate that neurogenic changes occur in patients with OSA. It is likely that no single mechanism can explain all the data concerning the alterations in motor unit potentials observed; rather, it is likely that our findings reflect factors predisposing to OSA combined with effects of snoring-induced vibration injury plus hypoxemia $(6,48,49)$. Clearly, structural neurogenic changes are present in patients with OSA. Further work is required to establish how these observed changes affect upper airway muscle function during sleep.

Author disclosures are available with the text of this article at www.atsjournals.org

Acknowledgment: The authors thank the staff at the Sleep Disorders Program, Brigham \& Women's Hospital, for assistance.

\section{References}

1. Eckert DJ, Saboisky JP, Jordan AS, Malhotra A. Upper airway myopathy is not important in the pathophysiology of obstructive sleep apnea. J Clin Sleep Med 2007;3:570-573.

2. Kimoff RJ. Upper airway myopathy is important in the pathophysiology of obstructive sleep apnea. J Clin Sleep Med 2007;3:567-569.

3. Kimoff RJ, Sforza E, Champagne V, Ofiara L, Gendron D. Upper airway sensation in snoring and obstructive sleep apnea. Am J Respir Crit Care Med 2001;164:250-255.

4. Nguyen AT, Jobin V, Payne R, Beauregard J, Naor N, Kimoff RJ. Laryngeal and velopharyngeal sensory impairment in obstructive sleep apnea. Sleep 2005;28:585-593.

5. Friberg D. Heavy snorer's disease: a progressive local neuropathy. Acta Otolaryngol 1999;119:925-933.

6. Mayer P, Dematteis M, Pépin JL, Wuyam B, Veale D, Vila A, Lévy P. Peripheral neuropathy in sleep apnea: a tissue marker of the severity of nocturnal desaturation. Am J Respir Crit Care Med 1999;159:213-219.

7. Series F, Cote C, Simoneau JA, Gelinas Y, St Pierre S, Leclerc J, Ferland R, Marc I. Physiologic, metabolic, and muscle fiber type characteristics of musculus uvulae in sleep apnea hypopnea syndrome and in snorers. J Clin Invest 1995;95:20-25.

8. Series F, Simoneau JA, St Pierre S. Muscle fiber area distribution of musculus uvulae in obstructive sleep apnea and non-apneic snorers. Int J Obes Relat Metab Disord 2000;24:410-415.

9. Carrera M, Barbe F, Sauleda J, Tomas M, Gomez C, Agusti AG. Patients with obstructive sleep apnea exhibit genioglossus dysfunction that is normalized after treatment with continuous positive airway pressure. Am J Respir Crit Care Med 1999;159:1960-1966.

10. Lindman R, Stal PS. Abnormal palatopharyngeal muscle morphology in sleep-disordered breathing. J Neurol Sci 2002;195:11-23.

11. Smirne S, Iannaccone S, Ferini-Strambi L, Comola M, Colombo E, Nemni R. Muscle fibre type and habitual snoring. Lancet 1991;337:597-599.

12. Ferini-Strambi LJ, Smirne S, Moz U, Sferrazza B, Iannaccone S. Muscle fibre type and obstructive sleep apnea. Sleep Res Online 1998;1:24-27.

13. Saboisky JP, Butler JE, McKenzie DK, Gorman RB, Trinder JA, White DP, Gandevia SC. Neural drive to human genioglossus in obstructive sleep apnoea. J Physiol 2007;585:135-146.

14. Mezzanotte WS, Tangel DJ, White DP. Waking genioglossal electromyogram in sleep apnea patients versus normal controls (a neuromuscular compensatory mechanism). J Clin Invest 1992;89:1571-1579.

15. Buchthal F. Electrophysiological signs of myopathy as related with muscle biopsy. Acta Neurol 1977;32:1-29.

16. Sandberg A, Hansson B, Stålberg E. Comparison between concentric needle EMG and macro EMG in patients with a history of polio. Clin Neurophysiol 1999;110:1900-1908.

17. Finsterer J, Fuglsang-Frederiksen A. Concentric-needle versus macro EMG. II. Detection of neuromuscular disorders. Clin Neurophysiol 2001;112:853-860

18. Nandedkar SD, Sanders DB, Stålberg EV. EMG of reinnervated motor units: a simulation study. Electroencephalogr Clin Neurophysiol 1988; 70:177-184

19. Svanborg E. Impact of obstructive apnea syndrome on upper airway respiratory muscles. Respir Physiol Neurobiol 2005;147:263-272.

20. Fuglsang-Frederiksen A, Scheel U, Buchthal F. Diagnostic yield of the analysis of the pattern of electrical activity of muscle and of individual motor unit potentials in neurogenic involvement. J Neurol Neurosurg Psychiatry 1977;40:544-554.

21. Nandedkar S, Stålberg E. Simulation of macro EMG motor unit potentials. Electroencephalogr Clin Neurophysiol 1983;56:52-62.

22. Stålberg E, Andreassen S, Falck B, Lang H, Rosenfalck A, Trojaborg W. Quantitative analysis of individual motor unit potentials: a proposition for standardized terminology and criteria for measurement. J Clin Neurophysiol 1986;3:313-348

23. Nandedkar SD, Stålberg E, Kim Y, Sanders DB, Anne A. Use of signal representation to identify abnormal motor unit potentials in macro EMG. IEEE Trans Biomed Eng 1984;31:220.

24. Jabre JF. Concentric macro electromyography. Muscle Nerve 1991;14: $820-825$

25. Stålberg E. Macro EMG, a new recording technique. J Neurol Neurosurg Psychiatry 1980;43:475-482.

26. Stålberg E. Macro electromyography: an update. Muscle Nerve 2011;44: 292-302. 
27. Dengler R, Konstanzer A, Kuther G, Hesse S, Wolf W, Struppler A Amyotrophic lateral sclerosis: macro-EMG and twitch forces of single motor units. Muscle Nerve 1990;13:545-550.

28. Vogt T, Nix WA, Pfeifer B. Relationship between electrical and mechanical properties of motor units. I Neurol Neurosurg Psychiatry 1990;53:331-334.

29. Jordan AS, Wellman A, Heinzer RC, Lo YL, Schory K, Dover L, Gautam S, Malhotra A, White DP. Mechanisms used to restore ventilation after partial upper airway collapse during sleep in humans. Thorax 2007;62:861-867.

30. Boyd JH, Petrof BJ, Hamid Q, Fraser R, Kimoff RJ. Upper airway muscle inflammation and denervation changes in obstructive sleep apnea. Am J Respir Crit Care Med 2004;170:541-546.

31. Friberg D, Ansved T, Borg K, Carlsson-Nordlander B, Larsson H, Svanborg E. Histological indications of a progressive snorers disease in an upper airway muscle. Am J Respir Crit Care Med 1998;157:586593.

32. Friberg D, Gazelius B, Hokfelt T, Nordlander B. Abnormal afferent nerve endings in the soft palatal mucosa of sleep apnoics and habitual snorers. Regul Pept 1997;71:29-36.

33. Friberg D, Gazelius B, Lindblad LE, Nordlander B. Habitual snorers and sleep apnoics have abnormal vascular reactions of the soft palatal mucosa on afferent nerve stimulation. Laryngoscope 1998;108:431436.

34. Ramchandren S, Gruis KL, Chervin RD, Lisabeth LD, Concannon M, Wolfe J, Albers JW, Brown DL. Hypoglossal nerve conduction findings in obstructive sleep apnea. Muscle Nerve 2010;42:257-261.

35. Guilleminault C, Huang YS, Kirisoglu C, Chan A. Is obstructive sleep apnea syndrome a neurological disorder? A continuous positive airway pressure follow-up study. Ann Neurol 2005;58:880-887.

36. Lim DC, Veasey SC. Neural injury in sleep apnea. Curr Neurol Neurosci Rep 2010;10:47-52.

37. Kirkness JP, Schwartz AR, Schneider H, Punjabi NM, Maly JJ, Laffan AM, McGinley BM, Magnuson T, Schweitzer M, Smith PL, et al. Contribution of male sex, age, and obesity to mechanical instability of the upper airway during sleep. $J$ Appl Physiol 2008;104:1618-1624.

38. Saboisky JP, Hamilton-Wright A, Nandedkar S, Stashuk DW, Carusona AL, Trinder JA, David WS, McSharry DG, Malhotra A. Examination of motor unit potentials in the genioglossus using automated decomposition based quantitative electromyography (DQEMG) [abstract]. Presented at the 31st Annual Meeting of the Australian Neuroscience Society, January 31-February 3, 2011, Auckland, New Zealand, 21:47.

39. Saboisky JP, Stashuk DW, Hamilton-Wright A, Nandedkar S, Carusona AL, Campana LM, Trinder JA, McSharry DG, David WS, Malhotra A. Automated decomposition based quantitative electromyography (DQEMG) in the examination of genioglossus motor unit potentials [abstract]. Am J Respir Crit Care Med 2011;183:A3687.

40. Saboisky JP, Jordan AS, Eckert DJ, White DP, Trinder JA, Nicholas CL, Gautam S, Malhotra A. Recruitment and rate-coding strategies of the human genioglossus muscle. J Appl Physiol 2010;109:19391949.

41. Nashed J, Hamilton-Wright A, Stashuk DW, Faris M, McLean L. Assessing motor deficits in compressive neuropathy using quantitative electromyography. J Neuroeng Rehabil 2010;7:39.

42. Stashuk DW. Decomposition and quantitative analysis of clinical electromyographic signals. Med Eng Phys 1999;21:389-404.

43. Sonoo M. New attempts to quantify concentric needle electromyography. Muscle Nerve 2002; Suppl 11:S98-S102.

44. Zalewska E, Hausmanowa-Petrusewicz I, Stålberg E. Modeling studies on irregular motor unit potentials. Clin Neurophysiol 2004;115:543-556.
45. Zalewska E, Hausmanowa-Petrusewicz I. Evaluation of MUAP shape irregularity-a new concept of quantification. IEEE Trans Biomed Eng 1995;42:616-620.

46. Stålberg E. Macroelectromyography in reinnervation. Muscle Nerve 1982;5:S135-S138.

47. Gan R, Jabre JF. The spectrum of concentric macro EMG correlations. II. Patients with diseases of muscle and nerve. Muscle Nerve 1992;15: 1085-1088.

48. Dahlin LB, Lundborg G. Vibration-induced hand problems: role of the peripheral nerves in the pathophysiology. Scand J Plast Reconstr Surg Hand Surg 2001;35:225-232.

49. Takeuchi T, Futatsuka M, Imanishi H, Yamada S. Pathological changes observed in the finger biopsy of patients with vibration-induced white finger. Scand J Work Environ Health 1986;12:280-283.

50. Jäghagen EL, Berggren D, Isberg A. Swallowing dysfunction related to snoring: a videoradiographic study. Acta Otolaryngol 2000;120:438443.

51. Vuono IM, Zanoteli E, Oliveira AS, Fujita RR, Pignatari SS, Pizarro GU, Pradelle-Hallinan ML, Moreira GA. Histological analysis of palatopharyngeal muscle from children with snoring and obstructive sleep apnea syndrome. Int J Pediatr Otorhinolaryngol 2007;71:283290.

52. Svanborg E. Upper airway nerve lesions in obstructive sleep apnea. Am J Respir Crit Care Med 2001;164:187-189.

53. Keenan KG, Farina D, Merletti R, Enoka RM. Amplitude cancellation reduces the size of motor unit potentials averaged from the surface EMG. J Appl Physiol 2006;100:1928-1937.

54. Stewart CR, Nandedkar SD, Massey JM, Gilchrist JM, Barkhaus PE, Sanders DB. Evaluation of an automatic method of measuring features of motor unit action potentials. Muscle Nerve 1989;12:141-148.

55. Conwit RA, Stashuk D, Tracy B, McHugh M, Brown WF, Metter EJ. The relationship of motor unit size, firing rate and force. Clin Neurophysiol 1999;110:1270-1275.

56. Woodson BT, Garancis JC, Toohill RJ. Histopathologic changes in snoring and obstructive sleep apnea syndrome. Laryngoscope 1991; 101:1318-1322.

57. Blumen MB, de La Sota AP, Quera-Salva MA, Frachet B, Chabolle F, Lofaso F. Tongue mechanical characteristics and genioglossus muscle EMG in obstructive sleep apnoea patients. Respir Physiol Neurobiol 2004;140:155-164

58. Eckert DJ, Lo YL, Saboisky JP, Jordan AS, White DP, Malhotra A. Sensori-motor function of the upper airway muscles and respiratory sensory processing in untreated obstructive sleep apnea. $J$ Appl Physiol 2011;111:1644-1653.

59. Finsterer J, Fuglsang-Frederiksen A, Mamoli B. Needle EMG of the tongue: motor unit action potential versus peak ratio analysis in limb and bulbar onset amyotrophic lateral sclerosis. J Neurol Neurosurg Psychiatry 1997;63:175-180.

60. Martić V, Podnar S. Reference data for quantitative motor unit potential analysis in the genioglossus muscle. Muscle Nerve 2008;38:939-940.

61. Fawcett PR, Johnson MA, Schofield IS. Comparison of electrophysiological and histochemical methods for assessing the spatial distribution of muscle fibres of a motor unit within muscle. J Neurol Sci 1985; 69:67-79.

62. Buchthal F, Kamieniecka Z. The diagnostic yield of quantified electromyography and quantified muscle biopsy in neuromuscular disorders. Muscle Nerve 1982;5:265-280.

63. Nandedkar SD, Barkhaus PE, Sanders DB, Stålberg EV. Analysis of amplitude and area of concentric needle EMG motor unit action potentials. Electroencephalogr Clin Neurophysiol 1988;69:561-567. 